\section{Mise en place de l'asymétrie droite-gauche : Notch et Nodal dans le noud embryonnaire}

Stéphane Vincent
École Normale Supérieure de

Lyon, Cnrs UMR 5161, 46,

allée d'Italie, 69364 Lyon

Cedex 07, France.

Stephane.Vincent@ens-

lyon.fr
> Un des degrés de complexité des vertébrés supérieurs est l'asymétrie de leurs viscères. La mise en place de cette polarité droite-gauche (DG), chez la souris en particulier, se manifeste par la spécification du côté gauche dans l'embryon avant le début de l'organogenèse. Quatre étapes clés gouvernent ce processus (pour revue, voir [1] et Figure 1): (1) le premier événement est l'induction d'un signal asymétrique au niveau du nœud embryonnaire (centre organisateur localisé à l'extrémité apicale de la ligne primitive et responsable de l'organisation du tronc) probablement via le déclenchement d'un courant (flux nodal) orienté de la droite vers la gauche et créé par la rotation des cils des cellules ventrales du nœud; (2) ce signal se propage ensuite vers le mésoderme de la lame latérale gauche (MLL gauche); et (3) y active de façon transitoire une série de gènes conservés chez les vertébrés; (4) les produits de ces gènes permettent la traduction de ce signal asymétrique en différences morphologiques pendant l'organogenèse.

Quelles que soient les différences dans la mise en place de l'axe DG parmi les vertébrés, le gène Nodal, codant pour un membre de la super-famille des TGF- $\beta$ (transforming growth factor $\beta$ ) occupe une place majeure et conservée. Nodal, connu depuis longtemps pour son rôle primordial dans la mise en place de l'axe antéro-postérieur, est à l'origine de la cascade génique qui spécifie le côté gauche. Chez la souris, Nodal est le premier gène à être exprimé de façon asymétrique tout d'abord dans le nœud embryonnaire et ensuite dans le MLL gauche.
Trois études publiées récemment [2-4] démontrent une fonction précoce insoupçonnée de la voie de signalisation Notch dans la mise en place de l'axe DG. La voie Notch, conservée chez les eucaryotes supérieurs, est connue pour son rôle dans la médiation de nombreuses interactions cellulaires au cours $d u$ développement et en particulier dans la compartimentalisation de domaines spécifiques de l'aile de drosophile ou au cours de la somitogenèse chez les vertébrés. La particularité de la voie Notch est d'être impliquée dans la communication entre deux cellules voisines via l'interaction entre un ligand membranaire (membre de la famille DSL; delta, ser- rata/jagged, Lag2) et un récepteur membranaire Notch. Cette interaction a pour conséquence le clivage de la partie cytoplasmique du récepteur Notch appelée NICD (Notch intracellular domain). Le NICD migre dans le noyau où il interagit avec les protéines CSL (CBFl/RBP$J \kappa, \operatorname{Su}(H), \operatorname{Lagl})$. Les protéines CSL sont des répresseurs transcriptionnels qui interagissent avec une séquence spécifique d'ADN. L'interaction avec NICD a pour conséquence la conversion de ces répresseurs en activateurs transcriptionnels, permettant ainsi l'activation des gènes cibles (pour revue, voir [5]). La mutation de certains gènes codant pour des produits impliqués dans la voie

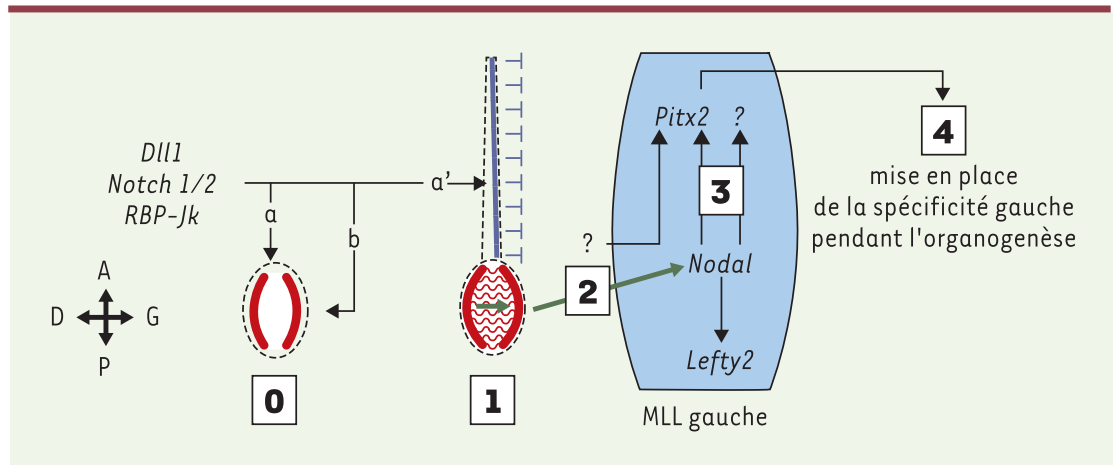

Figure 1. Mise en place de la polarité droite-gauche chez la souris. La spécification du côté gauche se fait en 4 étapes. 1. Cassure de la symétrie: le flux nodal produit par les cellules ventrales du nœud embryonnaire modifie l'expression symétrique de Nodal (schématisée en rouge) qui devient plus intense du côté gauche. 2. Transfert du signal asymétrique vers le mésoderme de la lame latérale gauche (MLL gauche) : la nature de ce signal n'est pas établie, mais les protéines Nodal et Fgf8 sont deux bons candidats. 3. Activation de l'expression asymétrique de gènes (Nodal, Lefty2, Pitx2...). 4. Conversion de l'expression génique en asymétrie morphologique pendant l'organogenèse. La voie Notch agit en amont de ces 4 étapes en activant l'expression de Nodal dans le nœud embryonnaire $(0, a)$. Cette voie semble intervenir également dans la morphogenèse du nœud $(0, b)$. De façon indirecte probablement via l'absence d'induction de Nodal dans le MML gauche, la voie Notch enclenche les mécanismes indispensables à la mise en place de la barrière de la ligne médiane ( 0 , $a^{\prime}$ : expression de Leftyl schématisée en bleu). 
Notch a pour conséquence la perturbation de la somitogenèse, mais elle affecte également la mise en place de l'axe DG [2-4]. La mutation du gène delta 1 (DIlI) codant pour un ligand de Notch, entraîne un phénotype similaire à celui qui est associé à la perte de fonction de Notchl dans un contexte Notch2 hypomorphe [4], ou de RBP-JK [4], effecteur principal du signal Notch. Au niveau moléculaire, l'expression des gènes spécifiques du côté gauche est affectée. En particulier, dans ces mutants, Nodal ne s'exprime pas dans le nœud (ce point est cependant controversé, G.K.Przemeck et al. ayant décrit que Nodal pouvait être détecté dans le nœud, ce que contestent $L$. Krebs et al. et A. Raya et al.) ; l'expression de l'antagoniste de Nodal, Leftyl, est absente dans le côté gauche du plancher neural suggérant l'absence de la barrière médiane. L'absence de Nodal dans le nœud des embryons $\mathrm{DllI}^{-/-}$explique l'absence d'activation des gènes en aval dans le MLL gauche, confirmant que l'expression de Nodal dans le nœud est cruciale pour la production du signal asymétrique, ce fait ayant déjà été démontré par l'analyse de deux allèles mutants du gène Nodal $[6,7]$. La présence de cellules anormales perturbant la forme du nœud suggère que la voie Notch agit également sur la morphogenèse du nœud [2]. La perte de l'expression de Leftyl pourrait être expliquée par la perturbation de la formation du plancher neural, structure dérivée du nœud embryonnaire, mais un travail récent suggère que c'est la conséquence de l'absence de Nodal dans le MLL gauche [8]. L'analyse de tous ces résultats et le fait que la perte de fonction de Dlll n'affecte pas le flux nodal [3] situent l'intervention de la voie Notch non pas dans la mise en place de la polarité $D G$, mais plutôt en amont, lors de l'activation de l'expression du gène clé Nodal (voir Figure 1, flèches $a$ et $b$ ).

Le contrôle de l'expression de Nodal dans le nœud embryonnaire est assuré par un élément régulateur spécifique qui a été caractérisé par transgenèse $[6,9,10]$ mais, jusqu'à présent, peu de données étaient disponibles quant à la nature des gènes impliqués dans l'activation de cet élément. J.Brennan et al. ont décrit des sites de fixation potentiels pour le facteur de transcription Foxa2 impliqué dans la mise en place du nœud mais sans montrer la réalité de cette interaction [6]. Les auteurs des deux études publiées dans Genes and Development ont montré la présence de deux sites de fixation pour $\mathrm{RBP}-\mathrm{J} \kappa$, actifs in vivo, démontrant le lien direct entre le contrôle de l'expression de Nodal et la voie de signalisation Notch.

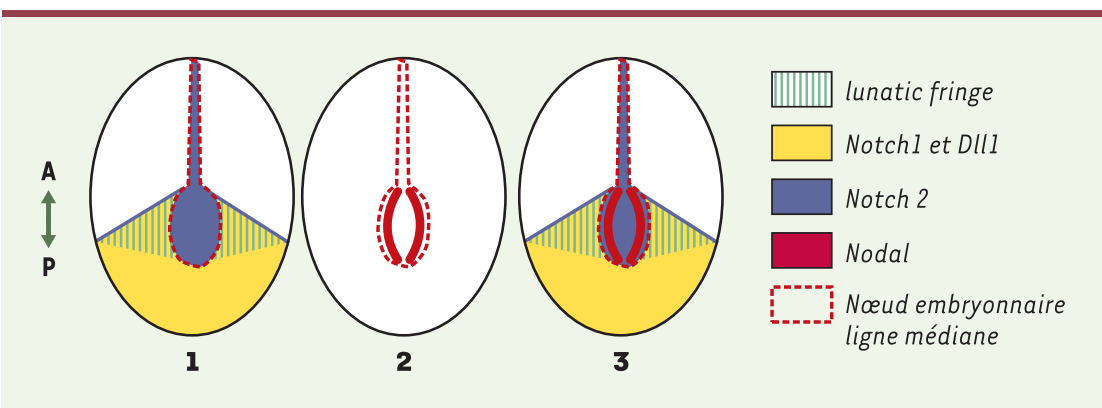

Figure 2. Profils d'expression des gènes Notch1/2, DII1, Lnfg et Nodal au moment du déclenchement de l'expression de Nodal dans le nœud embryonnaire. 1. Profils d'expression des gènes Notch1, Notch2, Dll1 et Lnfg à 7,5 jours post-coïtum. 2. Profils d'expression du gène Nodal au même stade de développement. 3. Superposition des profils d'expression montrant que le domaine d'expression de Nodal semble correspondre à l'intersection des profils d'expression de DIll, Notch1, Notch2 et Lnfg suggérant que ces 4 gènes participent à la régionalisation de l'expression de Nodal. Le gène codant pour RBP-JK, effecteur principal de la voie Notch, est exprimé de façon ubiquitaire. Les schémas représentent des embryons en vue ventrale.

Un autre aspect très intéressant de ces travaux est la démonstration que le gène Pitx2, effecteur de la spécification gauche et cible directe de Nodal, peut être induit en l'absence de Nodal, suggérant des mécanismes parallèles qui restent à décrire. Ceci remet en cause le dogme de l'unicité de Nodal en tant qu'activateur de la cascade génique spécifique du côté gauche dans le MLL.

Les résultats de ces travaux soulèvent une question intéressante. En effet, les profils d'expression des membres de la voie Notch sont très caractéristiques: Dlll et Notchl sont exprimés en bordure du nœud et dans le mésoderme postérieur, alors que Notch2 est exprimé dans le nœud ([24], Figure 2-1). Il est intéressant de noter que l'expression de Nodal n'est pas uniforme dans le nœud mais correspond à deux bandes de cellules en bordure du nœud (Figure 2-2). Comment expliquer alors que DIll, Notch1 et Notch2, qui sont exprimés de façon uniforme dans la bordure du nœud, y compris dans la partie postérieure, n'activent pas Nodal sur toute la bordure du nœud? Ceci pourrait suggérer que d'autres facteurs entrent en jeu. Une hypothèse séduisante mais non testée serait l'implication de lunatic fringe (Lngf) codant pour une glycosyltransférase. On sait en effet que $\operatorname{Lnfg}$ est capable de moduler l'interaction entre Notch et ses ligands; en particulier, chez la drosophile, Fringe potentialise l'interaction Notch-Delta au détriment de l'interaction Notch-Serrata. De façon remarquable, le profil d'expression de Lnfg dans la souris correspond à deux domaines latéraux partant de la bordure du nœud ([2-4], voir Figure 2-3). L'action de Lnfg pourrait être impliquée dans la régionalisation de l'activation de l'expression de Nodal dans le nœud embryonaire, le domaine d'expression de Nodal se superposant à l'intersection des domaines d'expression de Dlll, Notchl, Notch2 et Lnfg. La perte de fonction de Lnfg est létale à la naissance, mais aucun défaut de latéralité n'a été décrit jusqu'à présent [11]. II est possible que Lnfg ne soit pas un facteur essentiel, mais il 
serait intéressant d'étudier l'expression de Nodal dans les mutants Lnfg et de tester une éventuelle interaction génétique entre Nodal et Lnfg.

Si la philosophie de la mise en place de l'asymétrie DG est conservée chez les vertébrés, il y a des différences entre les espèces en particulier dans les mécanismes qui conduisent à l'expression de Nodal du côté gauche. A. Raya et al. ont montré que, chez le poisson zèbre, la voie Notch est aussi impliquée dans le contrôle de l'expression de Nodal et par conséquent dans la mise en place de l'asymétrie DG. II est donc réconfortant de constater que l'évolution a conservé des mécanismes directement en amont de la détermination de la polarité DG. Left-right asymmetry: Notch acts upstream of Nodal
RÉFÉRENCES

1. Hamada H, Meno C, Watanabe D, Saijoh Y. Establishment of vertebrate left-right asymmetry. Nat Rev Genet 2002; 3: 103-13.

2. Przemeck GK, Heinzmann U, Beckers J, Hrabe de Angelis M. Node and midline defects are associated with leftright development in Deltal mutant embryos. Development 2003; 130: 3-13.

3. Krebs LT, Iwai N, Nonaka S, et al. Notch signaling regulates left-right asymmetry determination by inducing Nodal expression. Genes Dev 2003; 17: 1207-12.
4. Raya A, Kawakami y, Rodriguez-Esteban C, et al. Notch activity induces Nodal expression and mediates the establishment of left-right asymmetry in vertebrate embryos. Genes Dev 2003; 17: 1213-8.

5. Mumm JS, Kopan R. Notch signaling: from the outside in. Dev Biol 2000; 228: 151-65.

6. Brennan J, Norris DP, Robertson $\varepsilon$ J. Nodal activity in the node governs leftright asymmetry. Genes Dev 2002; 16: 2339-44.

7. Saijoh Y, Oki S, Ohishi S, Hamada $\mathrm{H}$. Left-right patterning of the mouse lateral plate requires nodal produced in the node. Dev Biol 2003; 256: 160-72.

\section{NOUVELLE}

\section{La résistance du moustique Culex pipiens aux insecticides}

Mylène Weill, Olivier Duron, Pierryck Labbé, Arnaud Berthomieu, Michel Raymond

\author{
Institut des Sciences de \\ l'Évolution, Génétique et \\ Environnement, Université de \\ Montpellier II (CC 065), \\ 34095 Montpellier 05, \\ France. \\ weill@isem.isem. \\ univ-montp2.fr
}

> Le moustique Culex pipiens est présent en zones tropicales et tempérées. Sa nuisance due aux piqûres et aux maladies qu'il véhicule (filariose, fièvre du Nil...) a poussé l'homme à le combattre activement dans de nombreux pays à l'aide d'insecticides. Au cours des dernières décennies, Culex pipiens a développé des résistances à une grande variété d'insecticides (DDT, pyréthrinoïdes, carbamates, organophosphorés, toxines de Bacillus sphaericus, etc.).

La résistance aux insecticides organophosphorés (OP) représente un excellent modèle d'étude de l'adaptation à un nouvel environnement. Ces insecticides inhibent l'acétylcholinestérase, enzyme res- ponsable de l'hydrolyse de l'acétylcholine dans les synapses cholinergiques (Figure 1). Cette inhibition prolonge la durée de l'influx nerveux, ce qui conduit rapidement à la mort du moustique. Développer une résistance à ces toxiques implique donc de supprimer ou de diminuer l'inhibition de l'acétylcholinestérase des synapses. L'étude des différents phénotypes de résistance et des changements génétiques qui leur sont associés permet d'appréhender finement comment se fait cette adaptation et comment elle se modifie au cours du temps [1].

Il est important de comprendre que le moustique ne mute pas pour résister aux
8. Yamamoto M, Mine $N$, Mochida K, et al. Nodal signaling induces the midline barrier by activating Nodal expression in the lateral plate. Development 2003; 130: 1795-804.

9. Norris DP, Robertson EJ. Asymmetric and nodespecific nodal expression patterns are controlled by two distinct cis-acting regulatory elements. Genes Dev 1999; 13: 1575-88.

10. Adachi H, Saijoh Y, Mochida $K$, et al. Determination of left/right asymmetric expression of nodal by a left side-specific enhancer with sequence similarity to a lefty-2 enhancer. Genes Dev 1999; 13: 1589-600.

11. Zhang N, Gridley T. Defects in somite formation in lunatic fringe-deficient mice. Nature 1998; 394: 374-7. insecticides ! De très nombreuses mutations préexistent dans les immenses populations de moustiques. Lorsque des insecticides sont présents dans l'environnement, les moustiques qui ont des mutations favorables à leur survie se reproduisent et les transmettent ainsi à leurs descendants, alors que les moustiques sensibles aux toxiques meurent. Les mutations conférant aux moustiques la capacité de résister aux OP ne sont pas engendrées, mais sélectionnées par le milieu. Plus simplement, la fréquence des moustiques porteurs de ces mutations augmente dans un environnement toxique.

Les gènes impliqués dans la résistance aux OP chez Culex pipiens

Seuls trois locus sont responsables de résistances majeures, $\varepsilon s t-2, \varepsilon s t-3$ et 